\title{
Development of a Traceable Dynamic Force Calibration for Applications like Material Testing Machines
}

\author{
Rolf Kumme $^{1}$ \\ ${ }^{1}$ Physikalisch-Technische Bundesanstalt, Bundesallee 100, 38116 Braunschweig, Germany \\ rolf.kumme@ptb.de
}

\begin{abstract}
Summary:
This paper provides an overview of the EMPIR 18SIB08 ComTraForce project and describes a general concept for the development of a traceability chain for dynamic force calibration in applications like in the field of material testing machines.
\end{abstract}

Keywords: dynamic force, force traceability, EMPIR 18SIB08, ComTraForce

\begin{abstract}
Introduction
Forces are measured in many applications in industry and research and the traceability of the measurement of static forces is well established. However, in many mechanical applications such as material testing, the forces are time dependent. The vibration of the whole mechanical structure of a material testing machine results in a vibration of the force transducer and the mass distribution of the whole structure, which causes (in addition to the spring force related to the mechanical deformation) in inertia forces related to the acceleration of the mass distribution of the mechanical structure. A new joint research project within the scope of the European Metrology Research Programme (EMPIR) called 18SIB08 ComTraForce was launched on 1 September 2019 to develop methods and procedures for the measurement of static, continuous and dynamic forces with the aim of establishing comprehensive traceability for force metrology services [1].
\end{abstract}

\section{Concept of Traceability of Dynamic Forces}

The traceability of static forces is realized by means of force measuring devices used as force transfer standards and calibrated in accordance with established procedures such as ISO 376 [2], first to determine the calibration curve of a force measuring device in a national force standard machine or a force calibration machine traceable to the SI. In the second step, the calibrated force measuring devices are used to calibrate the force indication of the material testing machine in accordance with international standards such as ISO 7500-1 [3].

When applying a material testing machine, the force $F=F(t)$ is applied as a function of time to the specimen or the mechanical structure to be tested. This results in time-dependent deformations $x=x(t)$ of the force measuring device and a time-dependent deformation of the mechanical structure. The deformation of the force measuring devices takes place according to Hooke's law in first approximation proportional to the acting force:

$F=c \cdot x$, where $c$ is the spring stiffness.

If static forces are applied or if the force as a function of time increases or decreases with a constant velocity, the acting accelerations are zero. In this case, there is equilibrium between the applied force, which is generated by the testing machine, and the force acting on the specimen or the force acting on the transfer standard installed in the testing machine for calibration. In this case the acting forces are static or continuous forces. The spring forces in the force transducer of the testing machine and in the force transducer of the force transfer standard are equal; therefore, the testing machine force indication can be calibrated directly with the force transfer standard. For precise measurement, the creep effect of the machine transducer and of the force transfer standard must be considered to allow the uncertainty of the calibration to be reduced. Another influence that must be considered is the synchronisation of the measurement of the force measurement device in the testing machine and the force measurement with the force transfer standard.

In the case of dynamic testing in the testing machine in addition to the spring forces vibrations are generated which result in not negligible accelerations which generate additional inertia forces $F_{a}=m \cdot \ddot{x}$. As a consequence, the force indicated by the testing machine is not identical to the force acting on the specimen. To 
consider the effect of inertia forces between the machine transducer and the specimen, methods such as ASTM E467 and ISO 4965 take these effects into account $[4,5,6]$. These standards are good practice guides for verifying the machine performance under consideration of dynamic forces. However, such standards are only verification standards and not calibration standards for a dynamic calibration of the machine. The uncertainties of these methods are unknown and the standards lack uncertainty information.

It is the aim of the new EMPIR ComTraForce project to develop traceable dynamic calibration methods based on traceable force transfer standards for continuous and dynamic forces. This paper describes the basic concept for developing these methods.

Here, the main idea is to use force measuring devices with well known static and dynamic properties with a traceability to the $\mathrm{SI}$ as in the static calibration according to ISO 7500-1. To this end, in the first step, the static calibration curve of the force measuring device consisting of a force transducer, a measuring amplifier and an indication must be calibrated. In the second step, the dynamic properties must be determined.

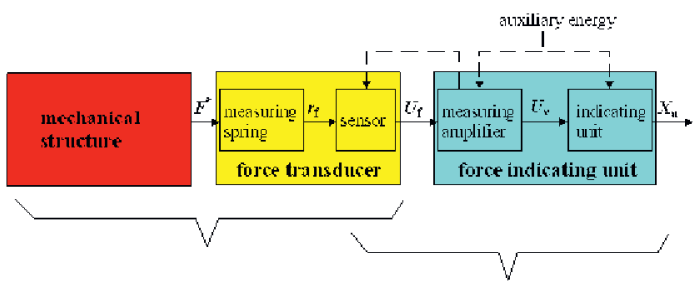

Fig. 1. Force measuring device in interaction with mechanical structure.

To establish traceability for dynamic forces in the field of material testing, it is necessary to select suitable force-measuring devices with a linear dependency between the indicated deflection and the acting force and a well-known frequency dependency of the force measuring device consisting of the force transducer and the measuring amplifier according to the block diagram in Fig. 1.

In addition to the static calibration and the frequency response of the force measuring device, the acting dynamic forces generated by the inertia force of the accelerated masses must also be considered. In addition to the calibration of the force measuring devices, it is necessary to determine the acceleration of the masses, which causes additional inertia forces between the machine force transducer and the specimen, as described in the model in Fig. 2. A traceability for dynamic forces in dynamic applications like in the field of material testing can be established by a combination of a traceable force and acceleration measurement devices. Each of these devices have to be calibrated and in addition to the uncertainty of the calibration of the force and acceleration measurement devices additional influences related to the application have to be taken into account.

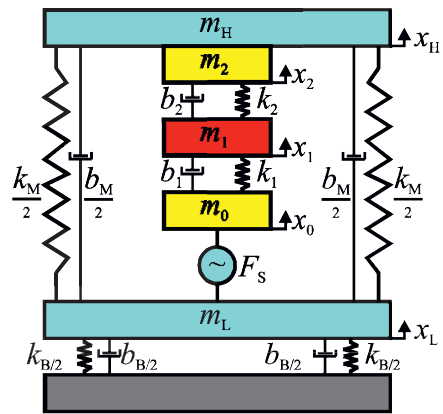

Fig. 2. Simplified model of a material testing machine.

\section{Summary}

In this paper, a concept for the development of a traceability chain for dynamic force calibration in dynamic applications like in the field of material testing machines is presented.

\section{Acknowledgements}

The author would like to acknowledge the funding of the joint research project 18 SIB08 ComTraForce. This project has received funding from the EMPIR programme, which is cofinanced by the European Union's Horizon 2020 research and innovation programme, and from EMPIR Participating States.

\section{References}

[1] 18SIB08 ComTraForce, Comprehensive traceability for force metrology services,

https://www.ptb.de/empir2019/comtraforce/home. html

[2] ISO 376: 2011 Metallic materials - Calibration of force-proving instruments used for the verification of uniaxial testing machines

[3] ISO 7500-1: 2018 Metallic materials - Calibration and verification of static uniaxial testing machines - Part 1: Tension/compression testing machines Calibration and verification of the forcemeasuring system

[4] ASTM E467 - 08(2014), Standard Practice for Verification of Constant Amplitude Dynamic Forces in an Axial Fatigue Testing System, ASTM International, West Conshohocken, PA, 2014, www.astm.org

[5] ISO 4965-1:2012 Metallic materials - Dynamic force calibration for uniaxial fatigue testing - Part 1: Testing systems

[6] ISO 4965-2:2012 Metallic materials - Dynamic force calibration for uniaxial fatigue testing - Part 2: Dynamic calibration device (DCD) instrumentation 